\title{
PD Detection on Power Cable with Peak-Correlation Method
}

\author{
Lihui Zhang, Yanda Li*, Mingjia Zhang, Hechen Liu and Yunpeng Liu \\ Hebei Provincial Key Laboratory of Power Transmission Equipment Security Defense, North China Electric Power University, \\ Baoding City, Hebei Province, 071003, China \\ ${ }^{*}$ Corresponding author
}

\begin{abstract}
Oscillating voltage is an effective method for PD detection in XLPE cable. In this paper, it mainly described the principle of oscillating waveform test system (OWTS) for PD detection and proposed the peak-correlation method for the location of partial discharge points. Then, an oscillating waveform test system for $10 \mathrm{kV}$ cable was established in the laboratory. It was used for the PD testing of a 500 meters long cable. In the end of the paper, the waveform characteristics of the direct pulse and reflect pulse were analyzed. With this feature, the problem caused by multi PDs in a single time window could be effectively solved which greatly improved the reliability and automation of the PD location algorithm. Experimental results show that oscillating voltage can effectively excite the generation of partial discharge and peak-correlation is a valid method for PD location with sufficient accuracy and reliability.
\end{abstract}

Keywords-component; oscillating voltage; cable; partial discharge; peak-correlation; location

\section{INTRODUCTION}

Nowadays, with the continuous improvement of the national economy, urban distribution network has rapid development. XLPE cable with its superior electrical performance and mechanical-physical properties has been widely applied $[1,2]$. To ensure the safety of the transmission network, domestic and foreign experts concentrate on partial discharge detection and localized discharge point of high voltage power cables, thus being one of the hot spots.

In order to facilitate the field test of capacitive electrical equipment, such as XLPE cables in recent years, Netherlands, Germany, Austria, the United States, Japan and other countries have proposed the use of oscillating power as a test power $[3,4]$ Oscillation voltage detection method overcomes the power frequency test power capacity, ultra-low frequency test and DC voltage test pressure time has poor equivalence, destructive influence and other shortcomings $[5,6]$, at home and abroad gradually gets widely used.

For the positioning of the partial discharge signal of the cable, the method of time-domain reflectometry (TDR) is usually used. The accuracy of the method depends on the accurate measurement of the time of arrival of cable forward wave and the reflected wave, through the time difference to locate. Usually the method of measuring TOA in time domain is threshold method, centroid method, peak method and correlation method. In the literature $[7,8]$, the above method is compared, and finally the peak method and the correlation method have high precision and stability. But in the actual test process, when the cable length is longer, partial discharge signal attenuation is serious, and a sufficiently low threshold must be set to successfully capture the partially discharged reflected wave signal, thus bringing a lot of noise interference; If the threshold is too high, it will not capture the reflected wave and cause positioning failure. The correlation method can extract the reflection waveform accurately by using the correlation between the front forward wave and the reflected wave in the case of strong noise, and then get the distance difference. However, due to the noise interference, the distance difference derived from the use of correlation may have a big deviation and inaccurate positioning. Considering the advantages and disadvantages of the above peak method and correlation method, this paper proposes combining two approaches. First, the correlation method is used to extract the reflected waveform, and then using the peak method for positioning to find the reflection peak. This method improves the anti-jamming ability of the algorithm, and ensures a high positioning accuracy, which can be effectively applied to engineering practice.

\section{PRinciple of Oscillating WAVE PARTial DISCHARGE DETECTION SYSTEM}

The oscillation wave detection technique is based on the LRC damping oscillation principle, the test processes are consists of two loops: a charging circuit and a damped oscillating discharge circuit. The cable is charged by a highvoltage DC power supply, when the charge is finished, close high voltage solid state switch, through the high-voltage reactor, high-voltage solid state switch and the test cable forms a damping oscillation voltage wave. Oscillation wave voltage test principle wiring diagram is shown in Figure I, the conversion of the charging circuit and the discharge circuit is realized by breaking the solid state switch $\mathrm{K}$. 


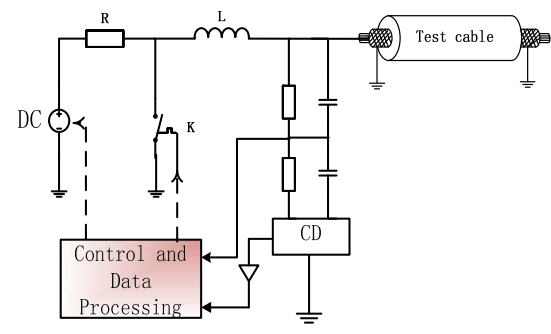

FIGURE I. PRINCIPLE CIRCUIT DIAGRAM OF THE OWTS PD DIAGNOSIS SYSTEM

The oscillation frequency can be calculated by the following equation:

$$
f=\frac{1}{2 \pi \sqrt{L C}}
$$

Where $\mathrm{L}$ is the inductance of the hollow reactor, $\mathrm{C}$ is the test cable capacitance, the oscillation frequency varies according to the cable length, generally between $50-500 \mathrm{~Hz}$ [9].

The sinusoidal voltage wave of the approximate frequency applied on the test piece excites the potential defect of the test product and forms a partial discharge signal.

The method of locating the local discharge point of the cable is the classic Time Domain Reflectometry (TDR) [1]. The principle is as follows:

Such as testing a length of cable, it is assumed that partial discharge occurs from the test end. The discharge pulse will travel along the cable in two opposite directions (as shown in Figure II). Set a pulse (forward wave) through t1 time to reach the test end, another pulse (reflected wave) at the end of the cable after the total reflection of t2 time to reach the test end. Depending on the time difference between the two pulses, the PD position can be located. The diagram is as follows:

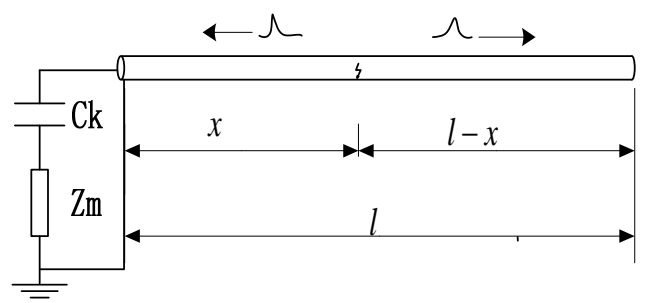

FIGURE II. PARTIAL DISCHARGE LOCATED BY TDR

In the figure: $\mathrm{Ck}$ is the high-voltage coupling capacitor, $\mathrm{Zm}$ is the detection impedance.

$$
\begin{gathered}
t_{1}=x / v \\
t_{2}=(2 l-x) / \mathrm{v}
\end{gathered}
$$

$$
x=l-v\left(t_{2}-t_{1}\right) / 2=l-\frac{v \Delta t}{2}
$$

In order to judge the local discharge position of the cable, it is necessary to accurately determine the time difference between the forward wave and the reflected wave, and then through the formula (4) to calculate the distance between the local discharge point and the measurement point. The consequence is obtained by calculating the arrival time (TOA) of the forward and reflected waves and calculating the difference between the two.

\section{CORRELATION-PEAK LOCAL DISCHARGE LOCATION METHOD}

\section{A. Correlation Partial Discharge Positioning}

Autocorrelation function, it is said to describe the value of the signal at a time and the value of another time depends on the relationship. Its' definition is:

$$
R_{x x}(\tau)=\lim _{T \rightarrow \infty} \frac{1}{T} \int_{0}^{T} x(t) x(t+\tau) d t
$$

Partial discharge signal is divided into two parts: one part of the direct arrival is the detection side and another part reaches the end of the cable and the total reflection of the final arrival of the detection end. The partial discharge signal is autocorrelation calculated: the maximum value is taken when $\tau=0$, and a sub-maximum peak is taken at $\tau \neq 0$. The time difference $\Delta t$ between the two is the time difference $\Delta t$ between the incident and reflected waves.

\section{B. Peak Method PD Positioning}

The peak method generally takes the highest point of the partial discharge pulse as the arrival time of the partial discharge signal. Figure III shows the $100 \%$ Peak of the partial discharge pulse signal. If the time of the preceding peak is tfpeak, the reflected wave is trpeak, then the time difference between the two is $\Delta \mathrm{t}=$ trpeak-tfpeak. 。

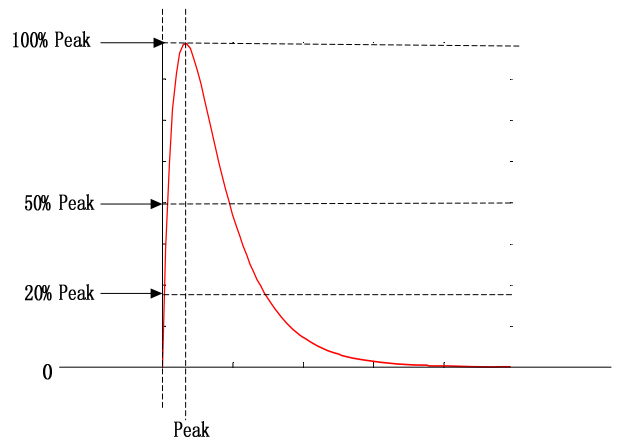

FIGURE III. PEAK OF THE SIGNAL 


\section{Correlation-peak Local Discharge Location Method}

The correlation algorithm can effectively extract the reflected waveform under high noise condition. But the higher noise may cause the positioning accuracy being not accurate. The main reason for this positioning deviation is that the noise signal interferes with the correlation of the partial discharge sequence, so that the relevant peak position changes. However, the resulting deviation stabilizes $\pm \delta \mathrm{N}$ ( $\delta \mathrm{N}$ is the relevant peak period). Therefore, this paper proposes the following method of partial discharge (PD) localization which is combined with correlation method and peak method.

To set the threshold according to the partial discharge signal, this threshold is only used to extract the forward wave waveform, as the forward wave amplitude is larger, it can set a higher threshold to ensure that all waveforms exceeding this threshold which are PD-signal forward wave. In addition, the higher threshold can also effectively reduce the impact of the interference signal on the stability and accuracy of the PD positioning.

Previously, the peak value of the traveling wave point $\mathrm{x}$ (k1), interception of partial discharge signal fragments $\{x(\mathrm{n} 1-$ $\mathrm{k}), \ldots, x(\mathrm{n} 1-\mathrm{k}+\mathrm{N}) \mid k$ is a constant, $\mathrm{N}$ is sequence length $\}$, $\mathrm{N}=2 \mathrm{l} / \mathrm{v} \times \mathrm{Fs}$, ( $l$ for the test cable length, $\mathrm{v}$ for the partial discharge signal in the cable propagation speed, as for the sampling rate). The forward and reflected waves are included in the length (time window), and contains as little as possible, and other partial discharge signal simplifies the positioning difficulty.

The localized discharge fragments are filtered and autocorrelation is performed. The distance difference $\mathrm{dN}$ between the forward and reflected waves is extracted, and the reflected wave position is $x\left(\mathrm{k}_{1}+\mathrm{dN}\right)$.

Finally, it should be find the reflected peak in the range $\mathrm{x}\left(\mathrm{k}_{1}+\Delta \mathrm{N} \pm \delta \mathrm{N}\right)$. The distance difference $\Delta \mathrm{N}(\Delta \mathrm{t}=\Delta \mathrm{N} / \mathrm{Fs})$ between the forward wave and the reflected wave is obtained.

\section{Oscillating Voltage PD Test}

The oscillating voltage test system was established and LabVIEW software was set up to be the measurement and analysis system. The $10 \mathrm{kV}$ single core cable was used to be the sample in the test and it's length was $500 \mathrm{~m}$. In order to generate partial discharge signal, the needle electrode was installed in joint parts to simulate defective cable.

The test circuit of OWTS for partial discharge was shown in Figure IV.

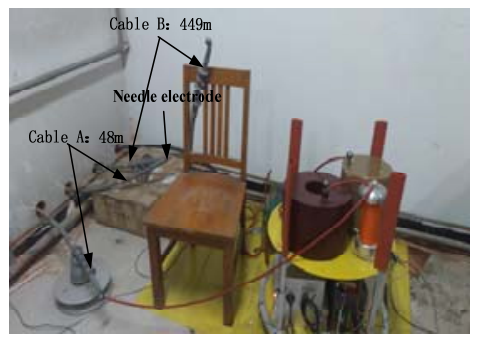

FIGURE IV. TEST CIRCUIT OF OWTS FOR PARTIAL DISCHARGE
Under this test condition, $6.5 \mathrm{kV}$ voltage value was the initial voltage of the test model, thus being PD phenomenon when the voltage rised to $6.5 \mathrm{kV}$. Ulteriorly, with voltage increasing, the partial discharge appearance was more obvious. The $7.5 \mathrm{kV}$ oscillation wave and the corresponding partial discharge signal was shown in Figure V.

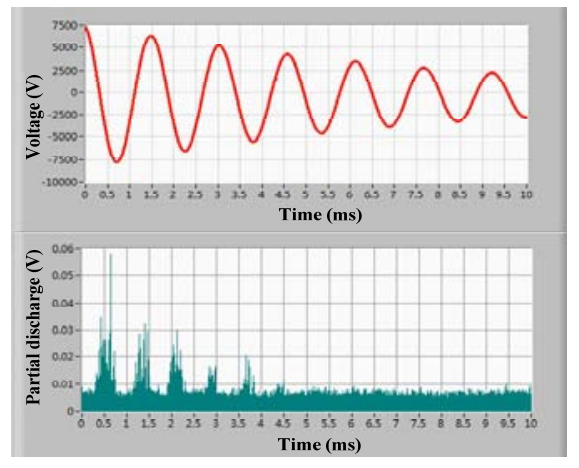

FIGURE V. OSCILLATING WAVEFORM AND PARTIAL DISCHARGE (7.5KV PEAK)

In this experiment condition (500m cable length), the oscillating frequency was $635.3 \mathrm{~Hz}$. The test result showed that the oscillating wave method had a good local discharge detection capability.

The peak-correlation method was applied for PD signal location and the signal threshold value was $0.015 \mathrm{~V}$. A large amount of interference signal would be imported to the system when the threshold was excessively low, which would also produce interference to the location analysis and affect the accuracy. Figure VI indicated the results of the partial discharge localization measurement by the self-correlation method. The result was aimed at the $464.66 \mathrm{~m}$ distance (relative to the distal end of the cable). It was clear that the location result was incorrect. However, the algorithm for the peakcorrelation method could automatically seek the reflected wave peaks in the vicinity of the blue point shown in Figure 4.3 (which was consistent with the preceding peak value type). The corrected location result was $502.21 \mathrm{~m}$ and the location error was $0.442 \%$.

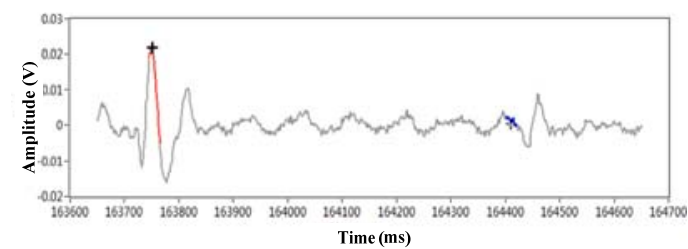

FIGURE VI. SELF-CORRELATION (464.66M)

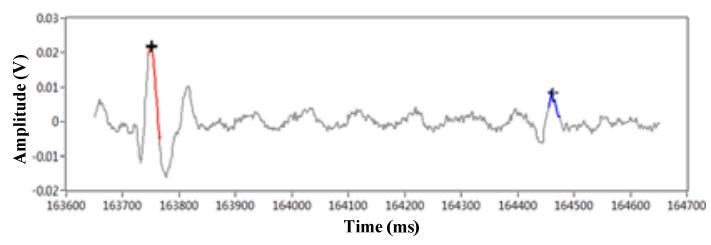

FIGURE VII. PEAK-CORRELATION (502.21M) 
The electric pulse at all voltage levels of the model was analyzed to obtain the partial discharge point corresponding to the forward waves and the reflected waves, and the partial discharge distribution mapping was displayed in Figure VIII.

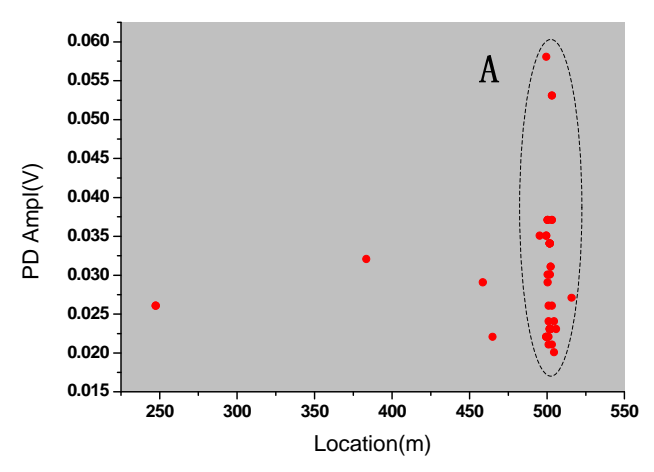

FIGURE VIII. PD MAPPING

\section{CONCLUSION}

In this paper, a partial discharge measurement system of oscillating wave cable was employed for the test in the laboratory. The insulating cardboard was used to be the discharge model and the effectiveness of the oscillating wave measurement system was explored previously. Experiment results indicated that it's highly effective to utilize oscillating voltage for partial discharge detection, and the method had many merits such as simple and convenient operation.

Moreover, the limitations of peak localization and selfcorrelation methods were analyzed respectively. It was proposed that the self-correlation algorithm was used to extract the reflected wave position primarily, and then the results were modified by the peak value method to collect the time difference between the forward and reflected waves. Finally, the partial discharge point was located precisely by the time domain reflection principle.

\section{ACKNOWLEDGMENTS}

This paper is supported by the Fundamental Research Funds for the Central Universities (2015MS91).

\section{REFERENCES}

[1] SUN Zhiming. Study and application about 10kV cable PD OWTS[D]. Beijing, China: North China Electric Power University, 2012.

[2] XIA Rong, ZHAO Jiankang, OUYANG Benhong, et al. Dielectric property detection of $110 \mathrm{kV}$ XLPE power cable using damped AC voltages[J]. High Voltage Engnineering, 2010, 36(07): 1735-1760.

[3] Gulski E, Wester F J, Smit J J, et al.Advanced partial discharge diagnostic of MV power cable system using oscillating wave test system[J]. IEEE Electrical Insulation Magazine, 2000, 16(2): 17-25.

[4] Gulski E, Smit J J, Seitz P, et al. PD measurements on-site using oscillating wave test system[C]//Conference Record of the 1998 IEEE International Symposium on Electrical Insulation. New York, USA:IEEE,1998: 420-423.

[5] LUO Junhua, FU Liwei, TAO Shili, et al. Comparing test of partial discharge on XLPE power cable at $0.1 \mathrm{~Hz}$ voltage and $50 \mathrm{~Hz}$ voltage[J]. High Voltage Engnineering, 2010, 36(8): 2000-2004.
[6] PENG Fadong, LIU Bin, Pang Xiaofeng, et al. Review of the equivalent between oscillating wave voltage and AC methods for XLPE cables withstand and partial discharge measurement[J]. High Voltage Apparatus, 2013, 49(07): 116-121.

[7] Firdaus A F, Mardiana R,Muyeen S M. Comparison of time-domain methods for two-end PD location in power cables[C]//2012 25th IEEE Canadian Conference on Electrical and Computer Engineering (CCECE). Piscataway, NJ, USA: IEEE,2012:6 pp.

[8] LING Fugen. Engneering applications of correlation and spectral analysis[M]. 1980: 121-152.

[9] Chen Z, Liu G, Chen C, et al. Test and Study of 10kV XLPE Power Cables' Typical Defects Based on Oscillating Wave Test System[C]//2010 IEEE International Symposium on Electrical Insulation (ISEI2010). Piscataway, NJ, USA: IEEE,2010:4 pp. 